\title{
Atypical presentation of ventricular tachycardia
}

\author{
Authors: Farhan Malik, ${ }^{A}$ ThinThin Khaing, ${ }^{B}$ Sanjay Adlakha ${ }^{C}$ and Thandar Aye ${ }^{D}$
}

\begin{abstract}
Cardiac syncope and epileptic seizure are two very similar presentations and difficult to differentiate without a proper history, physical examination and investigations. In a former study, 10 out of 22 episodes of induced ventricular tachycardia or fibrillation can result in stereotypical tonic-clonic movement with varied electroencephalography changes. We present a case which was diagnosed as ventricular tachycardia from seizure-like attack. It is to emphasise the importance of including ventricular tachycardia among other differential diagnoses of seizure-like activity in a patient with cardiovascular risks.
\end{abstract}

KEYWORDS: Ventricular tachycardia, epileptic seizure, cardiac syncope

DOI: $10.7861 /$ clinmed.2020-0046

\section{Introduction}

In a case of ventricular tachycardia, cardiac syncope can result in cerebral hypoxia and the patient can present with generalised tonic-clonic movement and loss of consciousness. This type of cardiac syncope carries a high risk of mortality but has a good prognosis if detected early. Hence, any patient with first seizure and high cardiovascular risks should be investigated for cardiac syncope using tests, such as telemetry in addition to electroencephalography (EEG) and brain scans. As in our case, we found out that the cause of seizure in this elderly man with high cardiovascular risks was ventricular tachycardia and it resulted from a triple vessel disease, which was corrected by performing a coronary artery bypass graft (CABG).

\section{Case presentation}

An 87-year-old man was admitted with an episode of collapse at home while in bed, witnessed by his son who described it as 'shaking while in bed and unresponsive with shallow breathing'. He had a pre-existing history of hypertension, hypothyroidism, peripheral vascular disease and progressively worsening cognitive

Authors: ${ }^{\text {A }}$ Clinical fellow, King's Mill Hospital, Sutton in Ashfield, UK; ${ }^{B}$ senior clinical fellow, King's Mill Hospital, Sutton in Ashfield, UK;

${ }^{C}$ respiratory consultant, King's Mill Hospital, Sutton in Ashfield, UK;

${ }^{D}$ consultant cardiologist, King's Mill Hospital, Sutton in Ashfield, UK and memory impairment over the last 6 months. He was found by his son in bed, unresponsive, followed by shaking of the left arm that lasted approximately 5 minutes, and then resolved spontaneously. There was no history of tongue biting, frothing, urinary or bowel incontinence. There was no postictal drowsiness but the patient had no recollection of events at the time of recovery after the seizure.

Upon assessment on admission to the hospital, his confusion screen including blood tests, computed tomography of the head and chest X-ray showed no obvious abnormalities. However, his electrocardiography (ECG) showed longstanding left bundle branch block with sinus bradycardia at a rate of 54 beats per minute.

On examination, he had a Glasgow coma scale of 14 because he was still confused as a result of the postictal phase. His pulse was regular with good volume. His chest was clear on auscultation and heart sounds were normal. There was no focal neurological deficit.

The patient was diagnosed with their first epileptic seizure and was planned to be started on anti-epileptic therapy if the patient had another episode of seizure attack. In order to rule out any underlying arrhythmia, it was decided to put the patient on telemetry which subsequently showed ventricular tachycardia (Fig 1). Echocardiography was carried out in order to diagnose the cause of ventricular tachycardia; it showed regional motion abnormality, overall moderate left-ventricular dysfunction, mild left-ventricular hypertrophy and moderate mitral regurgitation. However, there was no significant structural wall abnormality. The patient also had coronary angiography which showed right dominant coronary system with proximal right coronary artery with $100 \%$ stenosis (Fig 2a); severe left main stem disease revealing mild left anterior descending coronary artery stenosis of $50 \%$ (Fig 2b); and left circumflex obtuse marginal artery with $70 \%$ stenosis (Fig 2c). The patient was treated with a CABG with significant improvement of the patient's symptoms and absence of seizures with no requirement for anticonvulsant drugs.

\section{Discussion}

The history, clinical presentation, investigations and differentiation between syncope and seizure are very similar and usually very challenging in the elderly population. ${ }^{1,2}$ Transient loss of consciousness can result from both syncope and seizures but pathophysiology is quite different. Loss of consciousness due to syncope can result from reversible cerebral hypoperfusion. ${ }^{3,4}$ There are different types of syncopes which include reflex syncope, cardiac syncope, orthostatic hypotension and cerebrovascular syncope. ${ }^{1,3}$ 


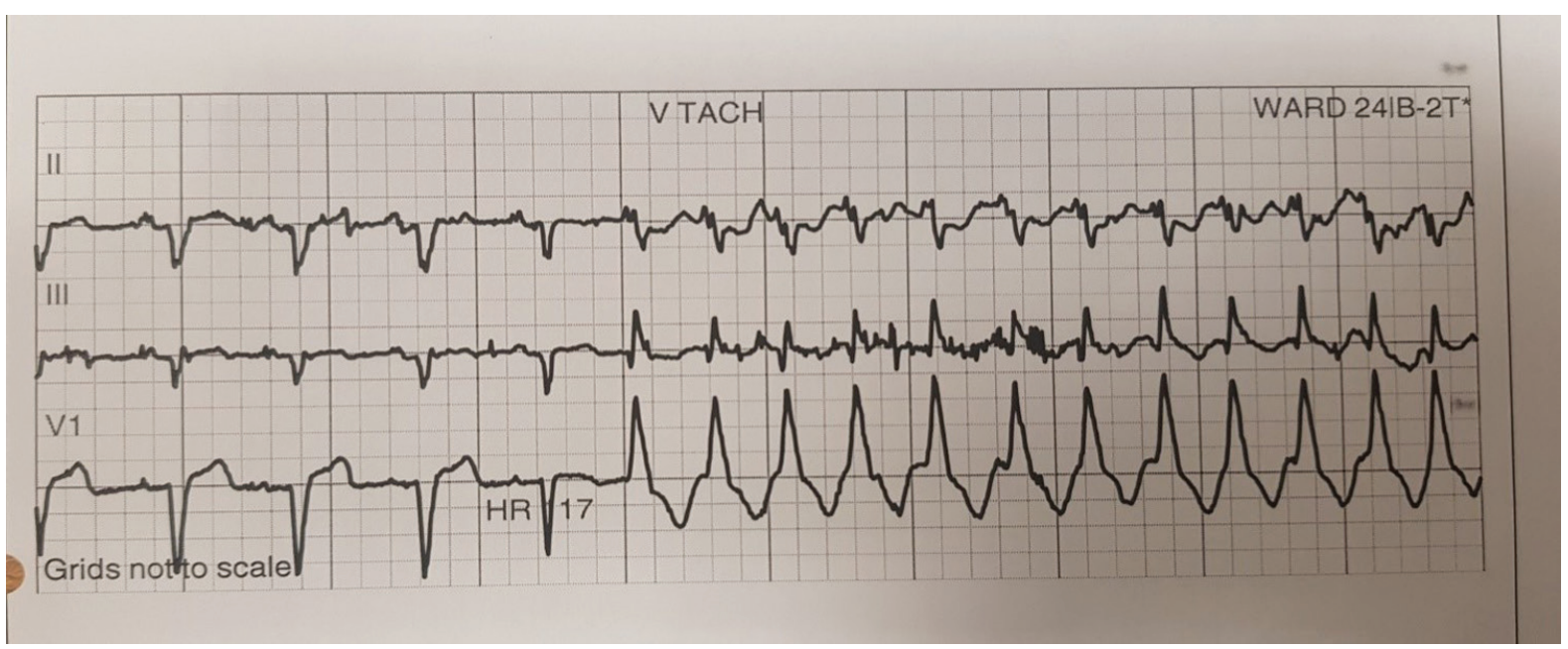

Fig 1. Telemetry showing ventricular tachycardia.

A combination of a careful detailed history from an eye-witness account, physical examination and ECG can result in up to $50 \%$ diagnostic yield for syncope, and these methodologies are also important in the accurate evaluation and diagnosis of seizure. ${ }^{1,5,6}$

In history taking, it is important to clarify the symptoms before, during and after the syncope. ${ }^{5}$ If there are postural symptoms such as prolonged standing, it is likely to be vasovagal or orthostatic syncope. If the attack occurs during defecation or micturition, it is likely to be micturition or defecation-related syncope. If there are exertional symptoms, cardiac syncope is most likely. ${ }^{7}$ However, syncope from cardiac arrhythmia can also occur at rest, eg when sitting or sleeping. ${ }^{7}$

The history of patient medication is crucial, as a recent change in the doses of certain medications such as antihypertensive drugs can result in syncope episode. The presence of symptoms suggestive of syncope and the lack of history of tongue bite, incontinence and prolonged confusion after an attack make epilepsy a more unlikely diagnosis. ${ }^{3}$

There are some additional histories which favour the diagnosis of syncope due to ventricular tachycardia and these include gender (male) and age of first onset of more than 35 years. The presence of prolonged sitting and standing, presyncope preceded by stress, recurrent headache, and fatigue lasting longer than a minute after syncope are in favour of vasovagal syncope. In addition to that, old age itself can serve as a peak period for seizure and epilepsy, and the incidence is higher in the elderly of more than 60 years old compared to the other age groups. ${ }^{8}$ These factors make an unexpectedly high frequency (20-30\%) of the misdiagnoses of epilepsy, wherein cardiovascular syncope was the most commonly misdiagnosed condition. ${ }^{1,2,9}$

The physical examination should be focused on lying and standing blood pressure, evidence of dehydration, signs of heart failure, presence of added heart sounds, rhythm abnormalities and any neurological deficit.

It is important to perform a wide range of tailored investigations to ascertain the correct diagnosis. To diagnose the exact cause of syncope, ECG alone can help up to $5 \%$ of cases and it is non-invasive and inexpensive. ${ }^{2,9}$ ECG findings such as bundle branch block, non-sustained ventricular tachycardia, previous myocardial infarction and left-ventricular hypertrophy
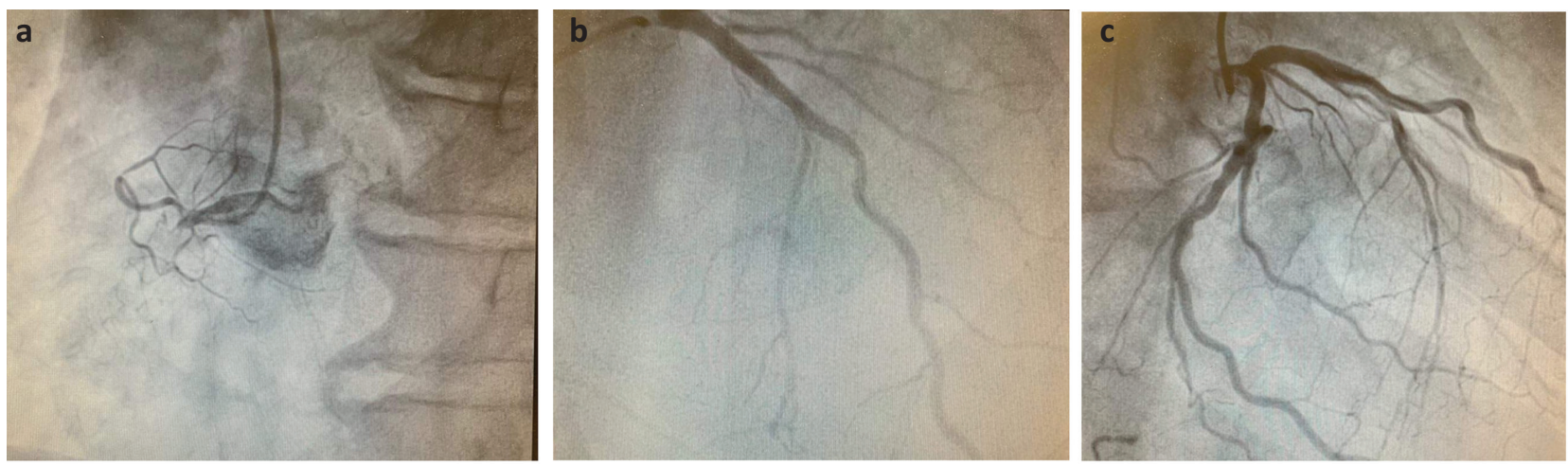

Fig 2. Coronary angiography. a) Left anterior-oblique view showing chronic total occlusion of proximal right coronary artery. b) Posterior-anterior cranial view showing severe left main stem stenosis (mild mid-left anterior descending disease). c) Posterior-anterior caudal view of severe left main stem stenosis and moderate obtuse marginal artery disease. 
may be an indication for further investigations. For these reasons, ECG is recommended in all syncope patients. ${ }^{1}$ For cases with no clues from ECG, there are other additional diagnostic tests such as head-up tilt table testing, Holter monitoring or telemetry, implantable loop recorder, echocardiography, ischaemia evaluation / stress testing, electrophysiological studies, carotid sinus massage, computed tomography / magnetic resonance imaging, EEG, simultaneous EEG and ECG, prolactin and creatinine kinase, and a diagnostic questionnaire for some undetermined cases. ${ }^{1,2,10}$

However, in a small percentage of patients with syncope or seizures, there are some conditions where loss of consciousness resulted from the interaction of syncope and seizure, and where they can provoke each other. Some cases have reported that cardiac arrhythmia can coincide with epileptic seizure as well as precede the attack of seizure, while some seizure attacks can also cause cardiac arrhythmias such as ictal sinus tachycardia, ventricular fibrillation, bradycardia and asystole, and vice versa. $^{1,9}$

In light of this, investigations aimed at both cardiac and neurological aspects are needed for the accurate evaluation, diagnosis and management of syncope and seizures.

\section{Conclusion}

It is important to consider ventricular tachycardia as a cause of first seizure in elderly patients especially with cardiovascular risks. So, in case of first seizure in elderly patients, the combination of careful history, thorough physical examination, epileptic and cardiac workup including 24-hour tape are vital, as revealed in the case of our patient where the cause of seizure was pinned down to the presence of ventricular tachycardia caused by ischaemic heart disease.

\section{References}

1 Rageti JS, Nai Q. Convulsive syncope induced by vebtricular arrythmia masquerading as epileptic seizure. Journal of Clinical Medicine Research 2016;8:610-5.

2 Josephson CB, Rahey S, Sadler RM. Neurocardiogenic syncope: frequency and consequences of its misdiagnosis as epilepsy. Can J Neurol Sci 2007:34:221-4.

3 van Dijk JG, Thijs RD, Benditt DG, Wieling W. A guide to disorders causing transient loss of consciousness: focus on syncope. Nat Rev Neurol 2009;5:438-48.

4 Yin $\mathrm{HC}$, Wu MN, Chen $\mathrm{CH}$, Haung P. Ventricular tachycardia manifested as tonic seizure. Epilepsy and behavior 2012;24:146-7.

5 Mayo Clinic. Ventricular tachycardia care at Mayo Clinic. Mayo Clinic. www.mayoclinic.org/diseases-conditions/ventricular-tachycardia/care-at-mayo-clinic/mac-20355147

6 Spikes GA, Liberty HG, Yates WH. Convulsive syncope due to rapid ventricular arrhythmias. Chest 1958;33:305-14.

7 Zaidi A, Fitzpatrick AP. Seizures and syncope: What's the difference? Cardiac Electrophysiology Review 2001;5:427-9.

8 Liu S, Yu W, Lü Y. The causes of new-onset epilepsy and seizures in the elderly. Neuropsychiatr Dis Treat 2016;12:1425-34.

9 Akhtar MJ. All seizures are not epilepsy: many have a cardiovascular cause. J Pak Med Assoc 2002;52:116-20.

10 Fattouch J, Di Bonaventura C, Strano S et al. Over-interpretation of electroclinical and neuroimaging findings in syncopes misdiagnosed as epileptic seizures. Epileptic Disord 2007;9:170-3.

Address for correspondence: Dr Farhan Malik, King's Mill Hospital, Mansfield Rd, Sutton-in-Ashfield NG17 4JL, UK. Email: malikfarhan001@gmail.com 